\title{
Effect of covalently bound RGD-peptide on PEEK on human osteoblasts
}

A.Schwitalla ${ }^{1}$, S.Herrmann ${ }^{1}$, W.-D.Müller ${ }^{1}$, M.Gabriel $^{2}$, T.Spintig ${ }^{1}$

Departement of Dental Research and Oral Biology, Universitätsmedizin Charité Berlin, Berlin, Germany 2 Department of Cardiothoracic and Vascular Surgery, Johannes Gutenberg University School of Medicine, Mainz, Germany

E-Mail: andreas.schwitalla@charite.de

\section{Background and Aim}

Due to its excellent properties, the high-performance biomaterial PEEK (polyetheretherketone) might represent a viable alternative to titanium as implant material. However, due to its bioinertness PEEK has to be surface-modified to achieve a sufficient osseointegration when being used as material for dental implants Therefore, a coating of the covalently bound peptide Arg-Gly-AspSer (RGD) on the PEEK surface might represent a possible solution to enhance cell adhesion onto PEEK.

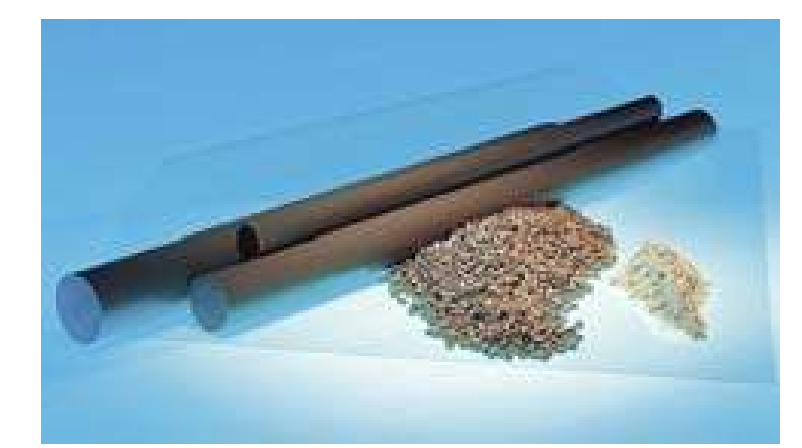

Fig.1 Different types of Polyetheretherketon (PEEK)

Aim: The Aim of the study was to evaluate the effects of a RGDpeptide coating on human osteoblasts in vitro compared to titanium and pure PEEK assuming that the covalently bound RGD-peptide enhances the cell adhesion and cell differentiation.

\section{Methods and Materials}

Twenty Disc-shaped specimens were fabricated from PEEK (Vestakeep ${ }^{\circledR}$ i2 R, Evonik Industries, Essen, Germany) and titanium $(n=10)$. The samples were polished up to 4000 grit Afterwards, ten of the PEEK samples were coated by RGDpeptide (RGD-PEEK) by means of Schiff base formation using ethylene diamine and following crosslinker-mediated fixation of the RGD-peptide, whereas ten samples were left untreated (PEEK). Surface roughness and -wettability were detected. Each sample was incubated at $37{ }^{\circ} \mathrm{C}$ with $1 \mathrm{ml}$ cell suspension containing 30,000 human osteoblasts. To evaluate the cell adhesion, cell differentiation and cell viability, the cell-covered area (CCA), the alkaline phosphatase (ALP) and the metabolization of alamar blue $(A B)$ were determined after 1 and 21 days.

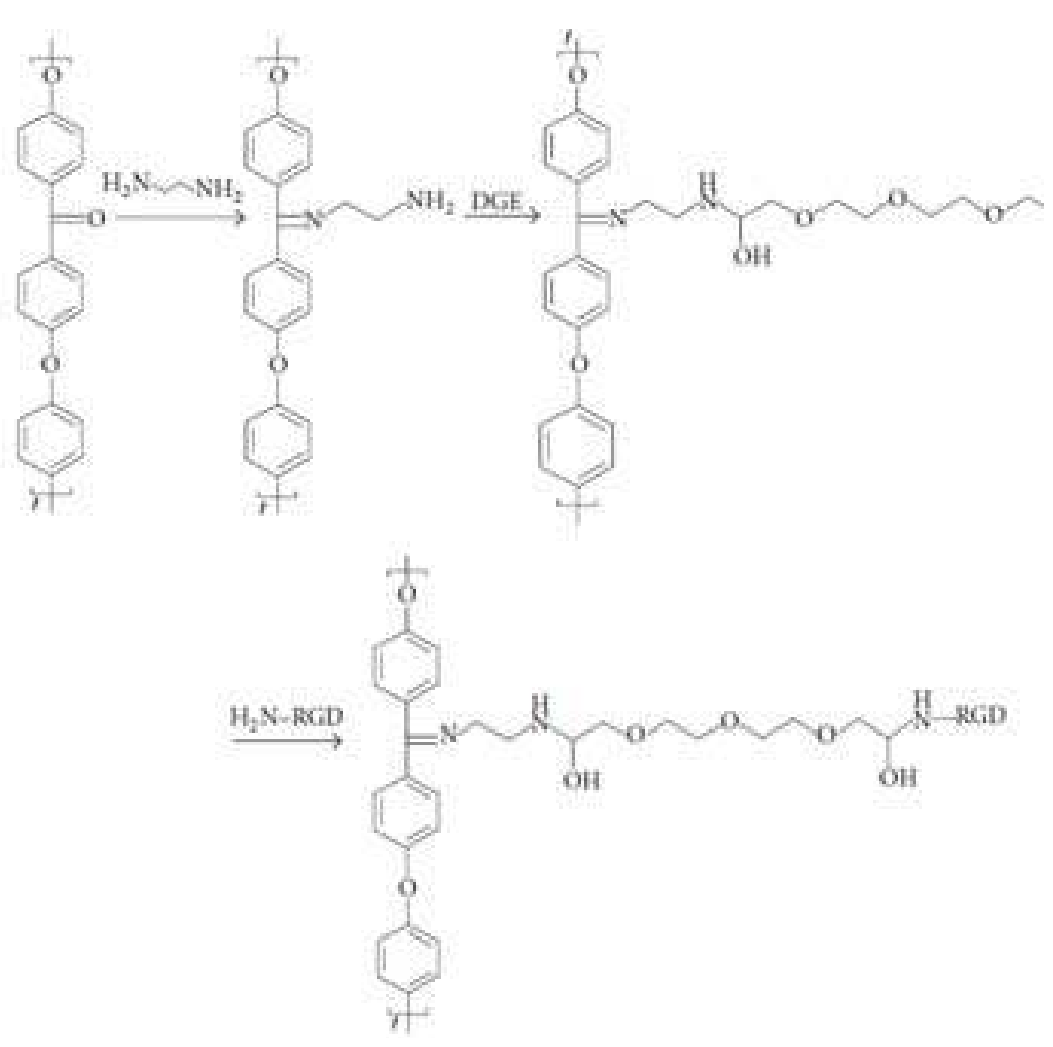

Fig.2 Reaction scheme for the cell-adhesive modification of PEEK. Surface activation was achieved via Schiff base formation using a diamine. Subsequent coupling of a diepoxide crosslinker afforded the covalent conjugation of the RGD-peptide [1]

\section{Results}

After 1 and 21 days, the PEEK-samples showed the highest CCA $(21.4 \pm 5.8 \%$ and $36.8 \pm 15.6 \%)$, followed by RGD-PEEK (14.8 $\pm 1.6 \%$ and $33.0 \pm 3.9 \%)$ and titanium $(7.7 \pm 4.7 \%$ and $31.0 \pm$ $18.4 \%$ ), whereas the increase in the CCA was significant for the RGD-PEEK samples. The differences in the CCA between the three groups were not significantly different.

After 1 day, the PEEK-samples showed the highest ALP extinction value $(0.2 \pm 0.01)$, followed by RGD-PEEK $(0.19 \pm$ $0.04)$ and titanium $(0.07 \pm 0.02)$. The ALP values increased after 21 days, whereas RGD-PEEK showed the highest extinction value $(0.51 \pm 0.16)$ which was significant different to day 1 followed by PEEK $(0.31 \pm 0.11)$ and titanium $(0.29 \pm 0.15)$. No significant difference was found between the groups 21 days.

The extinction values of $A B$ increased from day 1 to day 21 for all groups (PEEK: from $0.89 \pm 0.06$ to $1.23 \pm 0.06$; RGD-PEEK: from $0.87 \pm 0.06$ to $1.18 \pm 0.02$; titanium: from $0.95 \pm 0.2$ to $1.15 \pm$ $0.06)$.
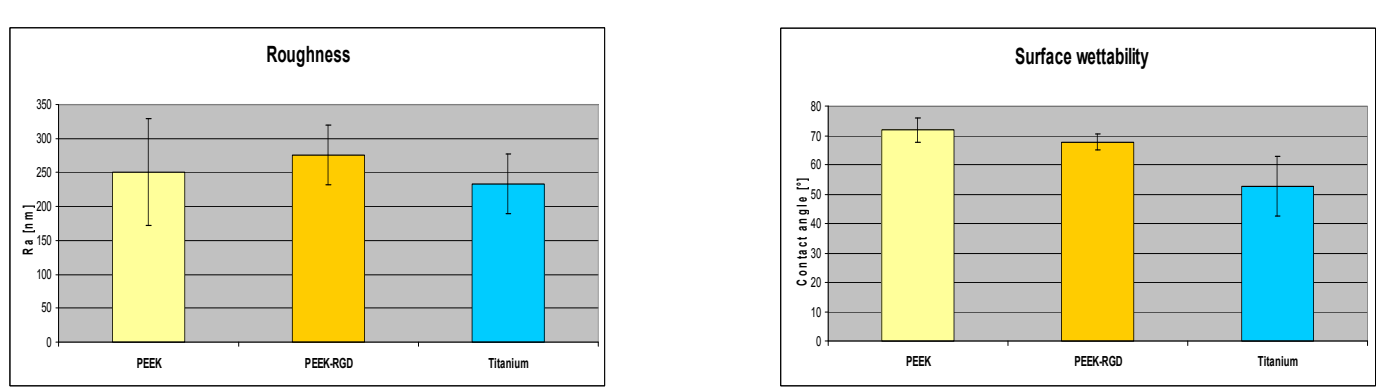

Fig.3 Roughness after surface modification Fig.4 Surface wettability

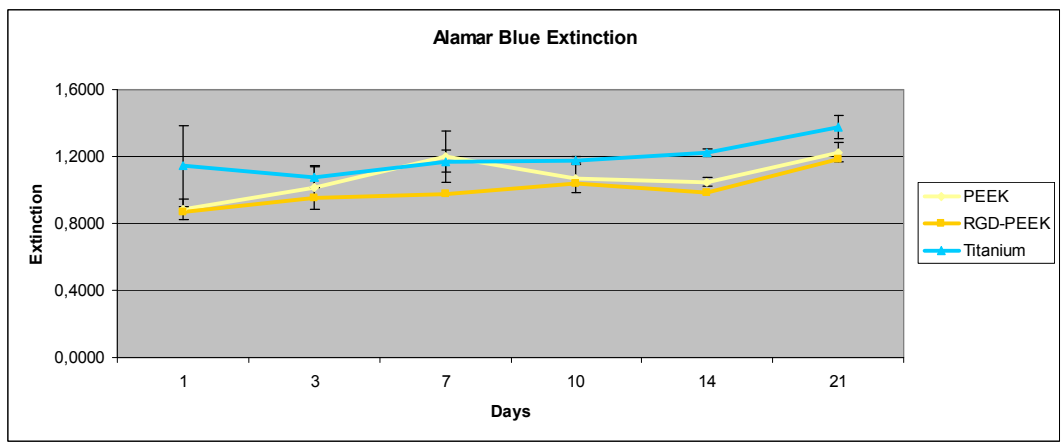

Fig.5 AlamarBlue Extinction from Day 1 to Day 21

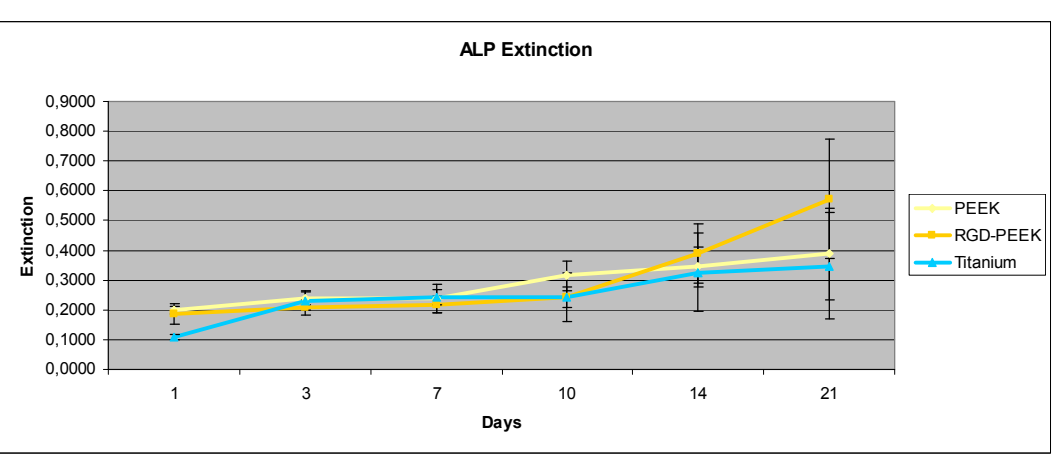

Fig.6 ALP Extinction from Day 1 to Day 21

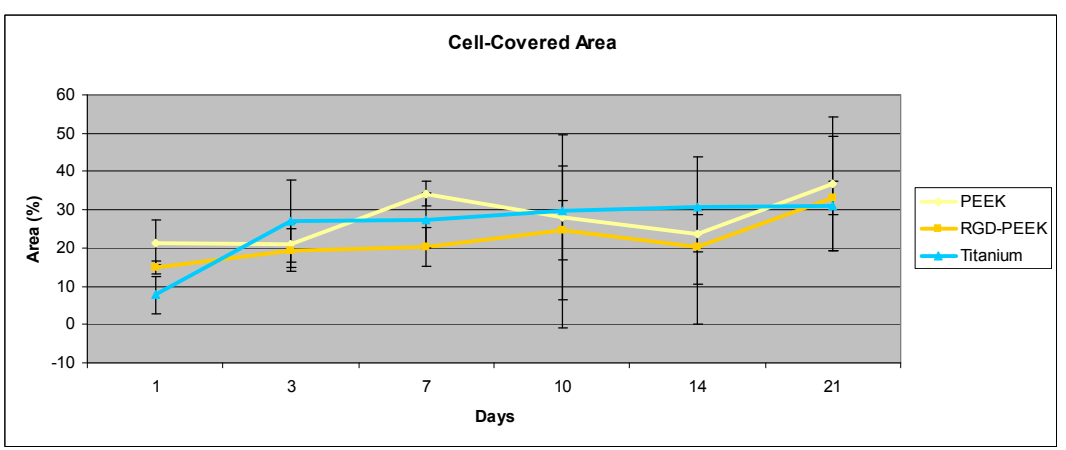

Fig.7 Cell-Covered Area from Day 1 to Day 21

\section{Conclusions}

Regarding the parameter ALP, which reflects the cell differentiation of the osteoblasts, and the CCA, representing the cell adhesion ability of the distinct surface, the covalently bound RGD-peptide as PEEK coating seems to represent one option to achieve a sufficient osseointegration of a PEEK dental implant. This has to be evaluated in vivo.

\section{References}

11 Covalent Grafting of the RGD-Peptide onto Polyetheretherketone Surfaces via Schiff Base Formation, The Scientific World Journal 